\title{
Warm SiO gas in molecular bullets associated with protostellar outflows
}

\author{
B. Nisini ${ }^{1}$, C. Codella ${ }^{2}$, T. Giannini ${ }^{1}$, J. Santiago Garcia ${ }^{3}$, J. S. Richer ${ }^{4}$, R. Bachiller ${ }^{3}$, and M. Tafalla ${ }^{3}$ \\ 1 INAF - Osservatorio Astronomico di Roma, via di Frascati 33, 00040 Monteporzio Catone, Italy \\ e-mail: nisini@oa-roma.inaf.it \\ 2 INAF - Istituto di Radioastronomia, Sezione di Firenze, Largo E. Fermi 5, 50125 Firenze, Italy \\ 3 Observatorio Astronómico Nacional (IGN), Alfonso XII 3, 28014 Madrid, Spain \\ 4 Cavendish Laboratory, J J Thomson Avenue, Cambridge CB3 0HE, UK
}

Received 17 May 2006 / Accepted 27 September 2006

ABSTRACT

\begin{abstract}
Context. We present the first $\mathrm{SiO}$ multiline analysis (from $J=2-1$ to $J=11-10$ ) of the molecular bullets along the outflows of the Class 0 sources L1448-mm and L1157-mm, obtained through observations with IRAM and JCMT.

Aims. We investigate the physical properties of different types of bullets, in order to explore their excitation conditions and constrain their formation and evolution.

Methods. We have computed the main physical parameters $\left(n_{\mathrm{H}_{2}}, T_{\mathrm{kin}}, N_{\mathrm{SiO}}\right)$ in each bullet and compared them with other tracers of warm and dense gas and with models for the $\mathrm{SiO}$ excitation in shocks.

Results. The bullets close to L1448-mm, associated with high velocity gas, have higher excitation conditions $\left(n_{\mathrm{H}_{2}} \sim 10^{6} \mathrm{~cm}^{-3}\right.$, $T \gtrsim 500 \mathrm{~K})$ than the L1157 bullets $\left(n_{\mathrm{H}_{2}} \sim 1-5 \times 10^{5} \mathrm{~cm}^{-3}, T \sim 100-300 \mathrm{~K}\right)$. In both the sources, there is clear evidence of the presence of velocity components having different excitation conditions, with the denser and/or warmer gas associated with the gas at the higher speed. In L1448 the bulk of the emission is due to the high-excitation and high velocity gas, while in L1157 most of the emission comes from the low excitation gas at ambient velocity. The observed velocity-averaged line ratios are well reproduced by shocks with speeds $v_{\mathrm{s}}$ larger than $\sim 30 \mathrm{~km} \mathrm{~s}^{-1}$ and densities $\sim 10^{5}-10^{6} \mathrm{~cm}^{-3}$. Plane-parallel shock models, however, fail to predict all the observed line profiles and in particular the very similar profiles shown by both low and high excitation lines.

Conclusions. The observations support the idea that the L1157 clumps are shock interaction events older than the L1448 bullets close to the driving source. In the latter objects, the velocity structure and the variations of physical parameters with the velocity very closely resemble those found in optical/IR jets near the protostar, suggesting that similar launching and excitation mechanisms are also at the origin of collimated jets seen at millimetre wavelengths.
\end{abstract}

Key words. ISM: jets and outflows - ISM: molecules - shock waves - submillimeter - ISM: individual objects: L1448-L1157 line: formation

\section{Introduction}

The mass-loss process starts during the earliest phases of star formation when protostars are still accreting mass (e.g. Richer et al. 2000, and reference therein). The stellar winds create jets that interact with the high-density clump hosting the newlyborn stars, forming shocks and driving molecular outflows. Fast $\left(\geq 100 \mathrm{~km} \mathrm{~s}^{-1}\right)$ collimated jets are observed both in optical emission of atomic lines and near infrared (NIR) $\mathrm{H}_{2}$ emission, showing excitation conditions with $T_{\mathrm{ex}}$ ranging from $\simeq 2000 \mathrm{~K}$ to more than $10000 \mathrm{~K}$. Also, extremely high velocity (EHV) jets have been seen in the form of narrow and high velocity $\mathrm{CO}$ and $\mathrm{SiO}$ molecular outflows in a handful of Class 0 sources (e.g. L1448-mm, HH211, IRAS04166, Guéth \& Guilloteau 1999; Tafalla et al. 2004). It is a matter of some debate whether the EHV molecular jet is the cold external layer of an embedded atomic jet, or whether the jet itself is intrinsically molecular. In particular, the EHV molecular jets show a knotty structure with regularly spaced clumps, the so-called molecular bullets, that are well-defined entities travelling at velocities of hundreds of $\mathrm{km} \mathrm{s}^{-1}$ (e.g. Bachiller et al. 1990; Chandler \& Richer 2001). The bullets are most likely associated with shocks formed by the outflow propagation into a homogeneous material and are consequently thought to be a direct manifestations of the episodic nature of outflows (Dutrey et al. 1997; Arce \& Goodman 2001, 2002). On the other hand, a few outflows driven by Class 0 objects (e.g. L1157-mm, NGC2264G, BHR71) show another type of molecular clump, characterised by lower velocities and by a rich chemistry: these clumps have strong emission due to molecular species that increase their abundance only when a shock-induced chemistry is active (Bourke et al. 1997; Bachiller et al. 2001). The origin of these chemically rich clumps is not clear: a possibility is that they are a consequence of a preexisting density enhancement and of its interaction with the passage of a shock (Viti et al. 2004).

However, the nature of both kinds of clumps and the possible relationships between them based on age, physical characteristics, and/or excitation conditions, are not yet understood. Detailed quantitative analysis of the EHV jets, bullets, and chemically rich clumps have been obtained so far with two standard outflow tracers such as $\mathrm{CO}$ and $\mathrm{SiO}$, but only with low frequency and consequently low excitation transitions. These transitions, however, are not suited to probe the warm gas components that may be associated with high-velocity and shocked gas. Indeed, sub-mm-wavelengths observations show that some molecular bullets are dense, $n_{\mathrm{H}_{2}} \sim 10^{6} \mathrm{~cm}^{-3}$, and 
warm, $T_{\text {ex }} \geq 100 \mathrm{~K}$ (Hatchell et al. 1997; Nisini et al. 2002; Palau et al. 2006).

In addition, ISO-LWS high- $J \mathrm{CO}$ and $\mathrm{H}_{2} \mathrm{O}$ low angular resolution observations seem to suggest that EHV jets are associated with temperatures in the 300-1500 K range (Giannini et al. 2001). Whether this warm gas represents a major component of the mass flux and consequently of the energetics of the mass loss from a YSO is still an open question.

In this paper, we investigate the nature of both the highvelocity bullets and chemically-rich clumps through the use of a multi-frequency analysis of the $\mathrm{SiO}$ emission. This molecule is the most typical tracer of high-density conditions and high velocity shocks, strong enough to release refractory elements in the gas phase (e.g. van Dishoeck \& Blake 1998). So far, SiO has been mainly observed at high velocities through its low rotational transitions $(J \leq 5)$, tracing relatively cold $(\leq 100 \mathrm{~K})$ gas (Martín-Pintado et al. 1992; Codella et al. 1999; Gibb et al. 2004).

The objects studied in this paper are the outflows L1448 and L1157, representative of flows associated with EHV jet-like bullets and lower velocity clumps respectively. Here we complement the low excitation spectra of $\mathrm{SiO} J=2-1,3-2$ and 5-4, with new observations of the $J=6-5,8-7$, and 11-10 emission at submillimetre wavelengths. High- $J$ SiO emission from $J=$ 5-4 to 11-10 has been observed in another typical EHV flow, HH211, by Nisini et al. (2002). Here we perform a detailed analysis of the full set of lines with the following aims: (i) to disentangle, through their excitation conditions, the warm gas components directly originating in the EHV jet from ambient and colder gas entrained by the jet itself; (ii) compare the spectra due to different transitions and thus investigate whether the excitation conditions vary with velocity, comparing the results with the predictions given by shock models; and (iii) estimate the type and strength of the shocks, and so place constraints on the origin of bullets and chemically rich clumps.

\section{Observations and results}

Observations were carried out with the IRAM 30-m telescope for the $\mathrm{SiO}$ transitions from $J=2-1$ to $6-5$, and with the James Clerk Maxwell Telescope (JCMT) for the SiO transitions from $J=8-7$ to 11-10. In Table 1, a list of the lines observed and their excitation energy is presented together with relevant observational parameters.

JCMT observations were performed during several nights in November 2001, and October and November 2002. We used the heterodyne receiver RxW for the $J=11-10$ and $J=10-9$ observations $\left(H P B W=12^{\prime \prime}\right)$, and RxB3 for the $J=8-7$ observations at $347 \mathrm{GHz}\left(H P B W=14^{\prime \prime}\right)$. The back-end was the digital autocorrelation spectrometer (DAS) with a bandwidth of $500 \mathrm{MHz}$. To convert the antenna temperature $\left(T_{\mathrm{A}}^{*}\right)$ into main-beam brightness temperatures, main-beam efficiencies $\eta_{\mathrm{MB}}$ equal to 0.55 and 0.4 were adopted for receivers B3 and W respectively. Pointing was checked on NGC7538 IRS1 and $\mathrm{W} 3(\mathrm{OH})$ and was good within about $3^{\prime \prime}$.

In L1448, we observed strips of 5 and 3 points in the $\mathrm{SiO} 8-7$ and 11-10 lines respectively, along the major axis of the outflow (PA 157.4) with a $10^{\prime \prime}$ spacing (see Fig. 1 ). The $(0,0)$ position of this strip corresponds to the exciting source of the outflow, L1448-mm $\left(\alpha_{2000}=03^{\mathrm{h}} 22^{\mathrm{m}} 34.3^{\mathrm{s}}, \delta_{2000}=30^{\mathrm{d}} 33^{\mathrm{m}} 35^{\mathrm{s}}\right)$.

The pointings along the strip covers the $\mathrm{CO}$ bullets $\mathrm{R} 1$ and R2 identified by Bachiller et al. (1990), resolved by the Guilloteau et al. (1992) interferometric observations in two
Table 1. Observed $\mathrm{SiO}$ transitions and instrumental setting.

\begin{tabular}{lcccccc}
\hline \hline Line & $\begin{array}{c}E_{\text {up }} \\
\mathrm{K}\end{array}$ & Instr. & $\begin{array}{c}\text { Freq. } \\
\mathrm{GHz}\end{array}$ & $\begin{array}{c}\text { Res. } \\
\mathrm{km} \mathrm{s}^{-1}\end{array}$ & $\begin{array}{c}H P B W \\
{ }^{\prime}\end{array}$ & $\eta_{B}$ \\
\hline $\mathrm{SiO} J=2-1$ & 6.2 & IRAM & 86.85 & 3.45 & 27.6 & 0.77 \\
$\mathrm{SiO} J=3-2$ & 12.5 & IRAM & 130.27 & 2.30 & 18.4 & 0.64 \\
$\mathrm{SiO} J=5-4$ & 31.2 & IRAM & 217.10 & 1.38 & 11.0 & 0.56 \\
$\mathrm{SiO} J=6-5$ & 43.7 & IRAM & 260.52 & 1.15 & 9.2 & 0.51 \\
$\mathrm{SiO} J=8-7$ & 75.0 & JCMT & 347.33 & 1.10 & 14 & 0.55 \\
$\mathrm{SiO} J=10-9$ & 114.5 & JCMT & 434.12 & 1.73 & 11 & 0.4 \\
$\mathrm{SiO} J=11-10$ & 137.4 & JCMT & 477.50 & 0.79 & 11 & 0.4 \\
\hline
\end{tabular}

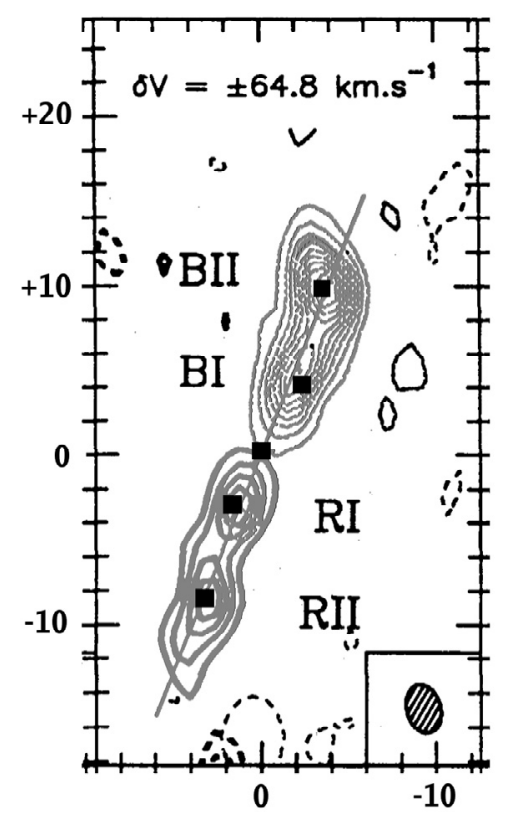

Fig. 1. The pointings adopted for our observations of the central region of L1448 are indicated by black squares over an interferometric velocity channel map at $64.8 \mathrm{~km} \mathrm{~s}^{-1}$ of the $\mathrm{SiO} 2-1$ transition taken from Guilloteau et al. (1997). Offsets (in arcsec) are with respect to L1448-mm.

distinct peaks each, named BI-BII and RI-RII and indicated in Fig. 1.

In the $(0,0)$ position a spectrum of the $\mathrm{SiO} 10-9$ transition was also obtained. Spectra of the pointings centered on the $\mathrm{mm}$ source and on the B1, R1 bullets are shown in Fig. 2. In addition to the central region, a $\mathrm{SiO} 8-7$ spectrum was obtained also in the south lobe of the outflow, at the position were a strong SiO 2-1 peak was observed by Dutrey et al. (1997; position R4 following Bachiller et al. 1990).

In L1157, a map in the SiO 8-7 line was acquired covering the strongest bullets identified by Bachiller et al. (2001), B0, B1 and B2 in the southern lobe of the outflow, and R0, $\mathrm{R} 1$ and $\mathrm{R}$ in the northern lobe (Fig. 3). Additional spectra of the SiO 10-9 transitions were obtained only in the B0, B1 and B2 positions. Figure 4 shows the spectra of the brightest B1 peak.

The JCMT observations were complemented by observations of $\mathrm{SiO}$ transitions at lower frequency obtained at nearby positions using the IRAM-30 m telescope (Santiago Garcia, Ph.D. Thesis). These observations were performed on the 14th June 2000. Heterodyne receivers A100, A230, D150, and D270 were tuned to observe all the lines simultaneously in wobbler-switching mode, with HPBW beams from $28^{\prime \prime}$ to $9^{\prime \prime}$, and a wobbler throw of $\sim 240^{\prime \prime}$. For all the transitions an 


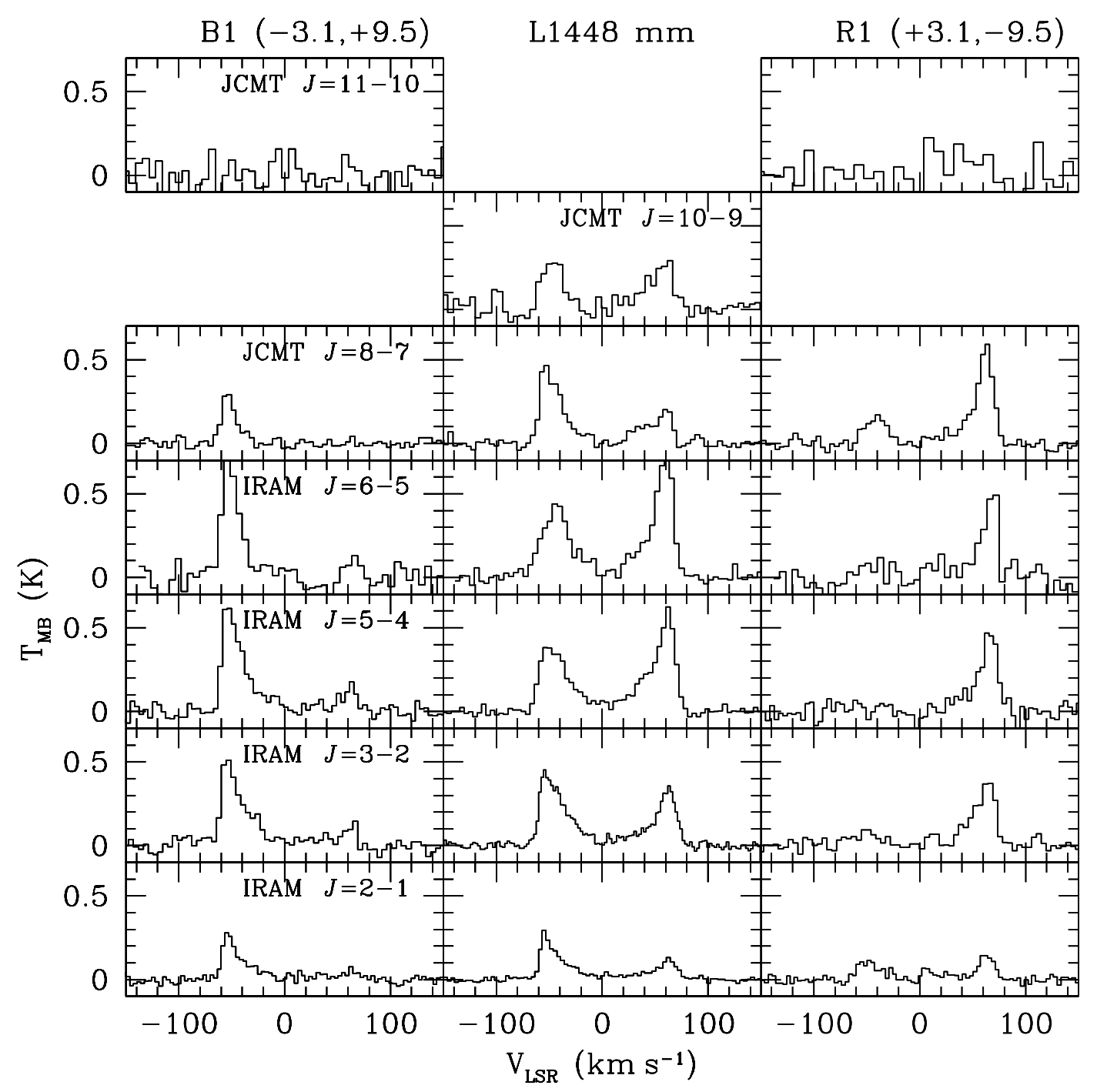

Fig. 2. Spectra of the $\mathrm{SiO}$ line emission taken in positions at the base of the L1448 outflow. Offsets (in arsec) of the B1 and R1 positions are with respect to the L1448-mm coordinates.

integration time of 4 min was accumulated in all the L1157 bullet spectra, and in the B1, R1 and R4 spectra in L1448, while the spectrum of the central L1448-mm position was integrated for $8 \mathrm{~min}$. Pointing was checked in nearby sources and found to be accurate within $2^{\prime \prime}$. System temperatures ranged from $\approx 166-$ $208 \mathrm{~K}$ for the $J=2-1$ line, to $\approx 1016-2180 \mathrm{~K}$ for the $J=6-$ 5 line. Spectra obtained with IRAM are also shown in Figs. 4 and 2, together with the JCMT spectra.

For both JCMT and IRAM observations, we have obtained the intensity integrated over the velocity channels, separating the blue- and red-shifted components. These values are listed in Tables 2 and 3 only for the positions that will be relevant for our analysis. The errors on the integrated intensities reported in these tables are the statistical errors due to thermal noise on the individual spectra. Calibration accuracy is about $10 \%$ for lines with $J \leq 8$, and $20 \%$ for the $J=10-9$ and 11-10 lines, and these uncertainties must be considered when estimating the total errors on observed quantities. In the L1157 flow, the coordinates of the beam centers in the JCMT observations do not always coincide with those of the IRAM observations, in which case we report in Table 3 the intensities obtained from the spectra closer in space. The angular difference of these spectra is typically of the order of $2-3^{\prime \prime}:$ in Fig. 3 , the different displacement of the IRAM and JCMT pointings is indicated.

Comparing the L1157 $\mathrm{SiO} J=8-7$ map with the $\mathrm{SiO} J=$ 3-2 and 5-4 maps in Bachiller et al. (2001), we see a similar brightness distribution: the strongest $\mathrm{SiO}$ emission is associated with the blue lobe, and in particular with bullet B1, the one most chemically active along the flow. In our map, we resolve the peak of the $\mathrm{B} 0$ bullet that remained unresolved in the 5-4 IRAM maps. We do not detect any $\mathrm{SiO}$ 8-7 emission in the $\mathrm{R} 1$ and R0 bullets. We also find a general agreement between the high- $J \mathrm{SiO}$ distribution and the distribution of the warm gas mapped through the pure rotational $\mathrm{H}_{2} \quad 0-0$ transitions by ISOCAM (Cabrit et al. 1999).

In L1448 the situation is different. The strongest high- $J$ lines are detected close to the millimetre source, in the B1 and R1 bullets, while the R4 bullet in the southern lobe, although very strong in $\mathrm{SiO} 2-1$, is much weaker in the high- $J$ lines. This behaviour was already pointed out by Bachiller et al. (1991) but this result is much clearer in our 8-7, 10-9 and 11-10 observations. In L1448 there is no $\mathrm{H}_{2}$ 0-0 map to compare the morphology of the $\mathrm{SiO}$ emission with the occurrence of warm gas; however, the R1 and B1 bullets are associated with the 
Table 2. Observed SiO intensities in L1448-mm.

\begin{tabular}{ccccccccc}
\hline \hline Offset $^{a}$ & Bullet & $J=2-1$ & $J=3-2$ & $J=5-4$ & $\begin{array}{c}J=6-5 \\
\int T_{\mathrm{MB}} d V^{b}\end{array}$ & $J=8-7$ & $J=10-9$ & $J=11-10$ \\
\hline$(-3.1,+9.5)$ & B1 & $6.3 \pm 0.3$ & $14.0 \pm 0.4$ & $15.8 \pm 0.7$ & $16.7 \pm 1.2$ & $5.3 \pm 0.5$ & $\ldots$ & $<1.2$ \\
& R1 & $1.9 \pm 0.3$ & $3.2 \pm 0.4$ & $3.8 \pm 0.7$ & $\ldots$ & $\ldots$ & $\ldots$ & $<1.2$ \\
\hline$(-2.3,+3.4)$ & B1 & $7.0 \pm 0.4$ & $13.0 \pm 0.4$ & $15.8 \pm 0.7$ & $19.0 \pm 1.2$ & $\ldots$ & $\ldots$ & $\ldots$ \\
& R1 & $3.7 \pm 0.4$ & $7.7 \pm 0.4$ & $11.2 \pm 0.7$ & $12.7 \pm 1.2$ & $\ldots$ & $\ldots$ & $\ldots$ \\
\hline$(0,0)$ & B1 & $6.6 \pm 0.2$ & $12.5 \pm 0.4$ & $13.0 \pm 0.4$ & $14.6 \pm 0.6$ & $12.0 \pm 0.5$ & $6.7 \pm 0.9$ & $<1.2$ \\
& R1 & $3.8 \pm 0.2$ & $9.4 \pm 0.4$ & $16.5 \pm 0.4$ & $18.8 \pm 0.6$ & $11.6 \pm 0.5$ & $8.7 \pm 0.9$ & $4.5 \pm 0.9$ \\
\hline$(+2.3,-3.4)$ & B1 & $5.1 \pm 0.4$ & $6.8 \pm 0.4$ & $6.4 \pm 0.7$ & $3.5 \pm 1.1$ & $\ldots$ & $\ldots$ & $\ldots$ \\
& R1 & $4.4 \pm 0.4$ & $9.4 \pm 0.4$ & $13.5 \pm 0.7$ & $20.6 \pm 1.1$ & $\ldots$ & $\ldots$ & $\ldots$ \\
\hline$(+3.1,-9.5)$ & B1 & $2.6 \pm 0.4$ & $2.6 \pm 0.5$ & $\ldots$ & $1.4 \pm 1.2$ & $4.0 \pm 0.4$ & $\ldots$ & $<1.2$ \\
& R1 & $4.0 \pm 0.4$ & $10.0 \pm 0.5$ & $11.1 \pm 0.7$ & $11.5 \pm 1.2$ & $13.3 \pm 0.4$ & $\ldots$ & $6.7 \pm 0.9$ \\
\hline$(+26,-128)$ & R4 & $13.1 \pm 0.3$ & $17.1 \pm 0.5$ & $10.7 \pm 0.5$ & $8.3 \pm 1.2$ & $3.1 \pm 0.4$ & & \\
\hline
\end{tabular}

${ }^{a}$ Offset in arcsec with respect to L1448-mm $\left(\alpha_{2000}=03^{\mathrm{h}} 22^{\mathrm{m}} 34.3^{\mathrm{s}}, \delta_{2000}=+30^{\mathrm{d}} 33^{\mathrm{m}} 35^{\mathrm{s}}\right) .{ }^{b}$ Flux errors refer to the rms noise in the individual spetra only. Total calibration uncertainty are of the order of $10 \%$ for the $J \leq 8$ lines and $20 \%$ for the $J=10$ and 11 lines.

region of the strongest high- $J \mathrm{CO}$ and $\mathrm{H}_{2} \mathrm{O}$ emission observed by ISO-LWS (Nisini et al. 2002).

The SiO 2-1 interferometric observations of Guilloteau et al. (1992) have shown that there is a velocity pattern along the $\mathrm{SiO}$ jet, with the velocity linearly increasing from BI-RI (at 3-4") to BI-RII (at $\sim 10^{\prime \prime}$ ). We have evidence of this behaviour in the shapes of the lines at different frequency, that are observed with different beam sizes. In the observations centered on the mm source, the blue and red-shifted emission peaks are located at velocities that decrease going from the $2-1\left(V_{\mathrm{LSR}}=\right.$ -55 and $+63 \mathrm{~km} \mathrm{~s}^{-1}$ for the B1 and R1 bullets respectively) to the 6-5 lines $\left(V_{\mathrm{LSR}}=-43.2\right.$ and $\left.+68.1 \mathrm{~km} \mathrm{~s}^{-1}\right)$, the latter having a beam size of only $9^{\prime \prime}$ and thus including only the slower BI-RI components.

\section{Analysis of the SiO excitation}

For a quantitative analysis of the $\mathrm{SiO}$ excitation employing ratios among different lines, we need to correct the derived intensities for the different beams adopted for the observations. In L1448 this correction can be significant, especially for the B1 and R1 knots that are located at $20^{\prime \prime}$ from the mm source. Each of these bullets is about $10^{\prime \prime}$ in length, but is not resolved in the transverse direction at a resolution of $2^{\prime \prime}$ (Guilloteau et al. 1992). Since we base our excitation analysis on the ratios between different lines, we have convolved all the observations along the strip to the beam of the $J=2-1$ transition, in order to obtain corrected ratios on the B1 and R1 bullets. In L1157 the problem seems to be less severe, since SiO IRAM maps of different transitions show that the bullets emission region is large even for the SiO 2-1 line taken with a 27" beam (Bachiller et al. 2001). In order to properly account for beam-filling effects, we have convolved the 8-7 data to the same resolution as the $2-1$ beam. We find that the peak temperature of the line decreases by a factor 1.3 in knot B2 to 2.2 in knot R. These changes in the peak temperature are consistent with a size of about $18-22$ arcsec in knots $\mathrm{R}, \mathrm{B} 0$, and $\mathrm{B} 1$ and around 30 arcsec for knot B2. In the analysis we have considered both the case of uncorrected line ratios and line ratios corrected assuming the above estimated sizes in order to evaluate the effect of beam dilution on the final results.

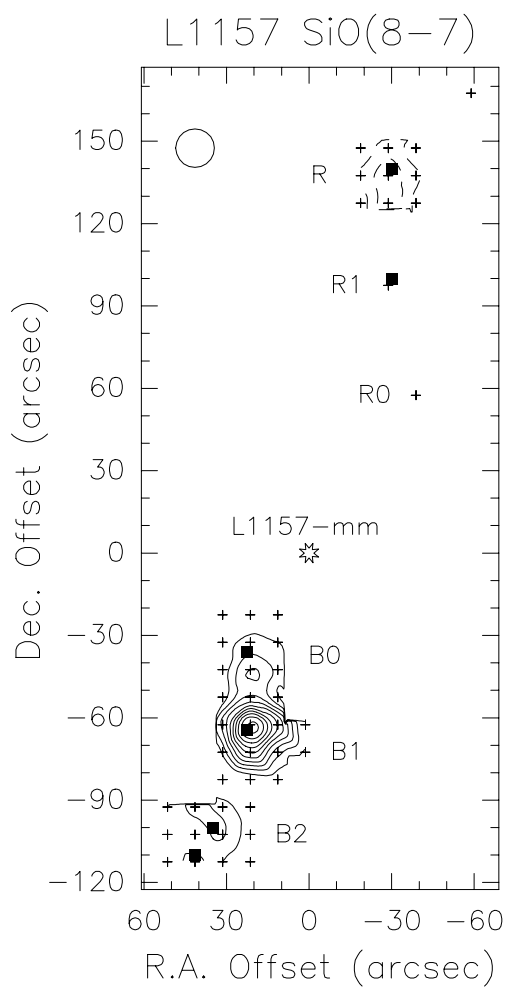

Fig. 3. Map of the $\mathrm{SiO} J=8-7$ integrated emission in the L1157 outflow. Offsets are with respect to L1157-mm. First contour and steps are $0.75 \mathrm{~K} \mathrm{~km} \mathrm{~s}^{-1}$ and $0.60 \mathrm{~K} \mathrm{~km} \mathrm{~s}^{-1}$ for the blue and red bullets, respectively (which correspond to $3 \sigma$ in each case). The circle represents the JCMT HPBW, while the small crosses indicate the observed positions. Filled squares indicate the pointings of the IRAM data considered in our analysis.

\subsection{Large Velocity Gradient analysis}

For the excitation analysis, we use an LVG model that considers the first $20 \mathrm{SiO}$ levels, with collision rates from Turner et al. (1992) up to $300 \mathrm{~K}$, plus the extrapolations given by Schöier et al. (2005) for higher temperatures (up to 2000 K). In Fig. 5 we plot the expected line brightness as a function of the rotational quantum number, for different values of the gas kinetic temperature and $\mathrm{H}_{2}$ density. While lines with $J_{\text {up }}<5$ do not strongly depend on the temperature but are sensitive to the density, high temperatures regimes $(T>100 \mathrm{~K})$ can be distinguished 
Table 3. Observed SiO intensities in L1157.

\begin{tabular}{|c|c|c|c|c|c|c|c|}
\hline Offset $^{a}$ & Bullet & $J=2-1$ & $J=3-2$ & $\begin{array}{r}J=5-4 \\
\int T_{\mathrm{ME}}\end{array}$ & $\begin{aligned} J & =6-5 \\
V^{b} & \end{aligned}$ & $J=8-7$ & $J=10-9$ \\
\hline$(-30,+100)$ & R1 & $4.7 \pm 0.2$ & $4.7 \pm 0.4$ & $<0.5$ & $<0.7$ & ... & $\ldots$ \\
\hline $\begin{array}{c}(-30,+140) \\
(-28.5,+137.5)\end{array}$ & $\mathrm{R}$ & $10.1 \pm 0.2$ & $13.4 \pm 0.4$ & $7.8 \pm 0.9$ & $2.0 \pm 1.2$ & $\begin{array}{c}\cdots \\
1.7 \pm 0.3\end{array}$ & $\begin{array}{l}\ldots \\
\ldots\end{array}$ \\
\hline $\begin{array}{l}(+22.5,-64.5) \\
(+21.1,-62.5) \\
(+21.1,-72.5) \\
\end{array}$ & $\overline{\mathrm{B} 1}$ & $19.3 \pm 0.3$ & $28.9 \pm 0.4$ & $24.9 \pm 0.9$ & $18.8 \pm 1.2$ & $\begin{array}{l}8.7 \pm 0.4 \\
4.6 \pm 0.4\end{array}$ & $\begin{array}{c}2.6 \pm 0.6 \\
\ldots \\
\end{array}$ \\
\hline $\begin{array}{c}(+22.5,-36) \\
(+21.1,-42.5) \\
\end{array}$ & B0 & $7.4 \pm 0.3$ & $9.5 \pm 0.4$ & $6.2 \pm 0.7$ & $4.4 \pm 1.2$ & $2.5 \pm 0.3$ & $<0.8$ \\
\hline $\begin{array}{c}(+35,-100) \\
(+31.1,-102.5) \\
(+41.1,-102.5)\end{array}$ & B2 & $12.7 \pm 0.3$ & $14.4 \pm 0.4$ & $6.5 \pm 0.7$ & $4.1 \pm 1.2$ & $\begin{array}{l}1.5 \pm 0.3 \\
1.8 \pm 0.3\end{array}$ & $\begin{array}{c}\cdots \\
\cdots \\
<1\end{array}$ \\
\hline $\begin{array}{c}+41.5,-110) \\
(+41.1,-112.5) \\
\end{array}$ & $\overline{\mathrm{B} 2}$ & $12.5 \pm 0.3$ & $15.2 \pm 0.4$ & $8.6 \pm 0.7$ & $5.0 \pm 1.2$ & $1.7 \pm 0.3$ & $\ldots$ \\
\hline
\end{tabular}

${ }^{a}$ Offset in arcsec with respect to L1157-mm, $\left(\alpha_{2000}=20^{\mathrm{h}} 39^{\mathrm{m}} 06.2^{\mathrm{s}}, \delta_{2000}=68^{\mathrm{d}} 02^{\mathrm{m}} 15.9^{\mathrm{s}}\right) .{ }^{b}$ Flux errors refer to the rms noise in the individual spetra only. Total calibration uncertainty are of the order of $10 \%$ for the $J \leq 8$ lines and $20 \%$ for the $J=10$ and 11 lines.

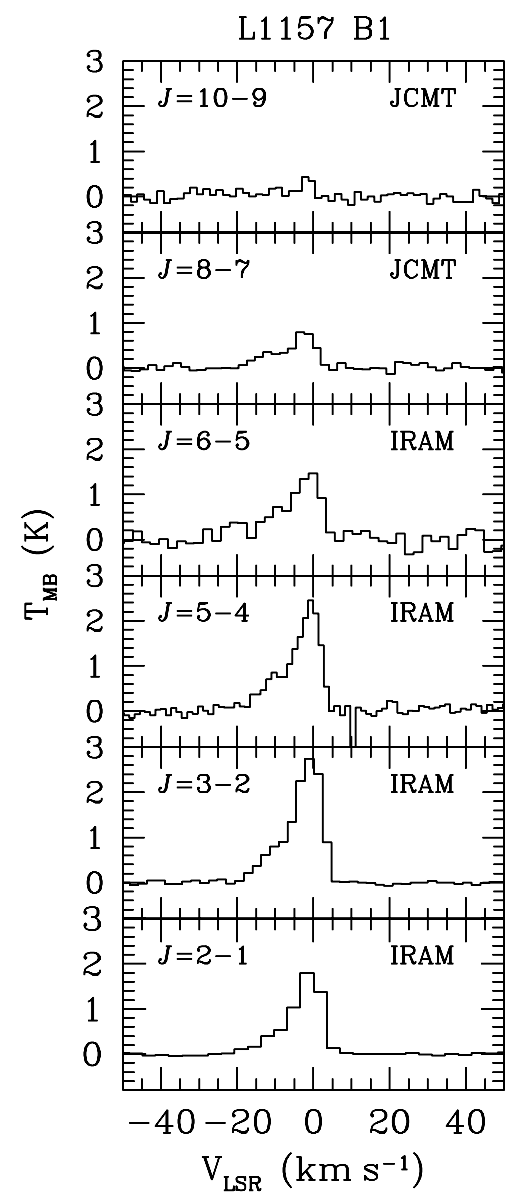

Fig. 4. Observed $\mathrm{SiO}$ spectra towards the $\mathrm{L} 1157 \mathrm{~B} 1$ position.

from low temperature gas only through high- $J(J \geq 5)$ lines. The plots also show that the lines become insensitive to the temperature for $T \gtrsim 500 \mathrm{~K}$. These plots have been obtained using a ratio between column density and velocity dispersion $\left(N_{\mathrm{SiO}} / \Delta V\right)$ of $10^{13} \mathrm{~cm}^{-2} \mathrm{~km}^{-1} \mathrm{~s}$. In this specific case, the maximum line opacity is $\sim 0.2$. Opacity effects start to become significant, affecting the line ratios up to the point at which the lines become insensitive to the $\mathrm{SiO}$ abundance, for $N_{\mathrm{SiO}} / \Delta V \sim 5 \times 10^{13} \mathrm{~cm}^{-2} \mathrm{~km}^{-1} \mathrm{~s}$, i.e. for column densities of the order of $5 \times 10^{14} \mathrm{~cm}^{-2}$ in gas

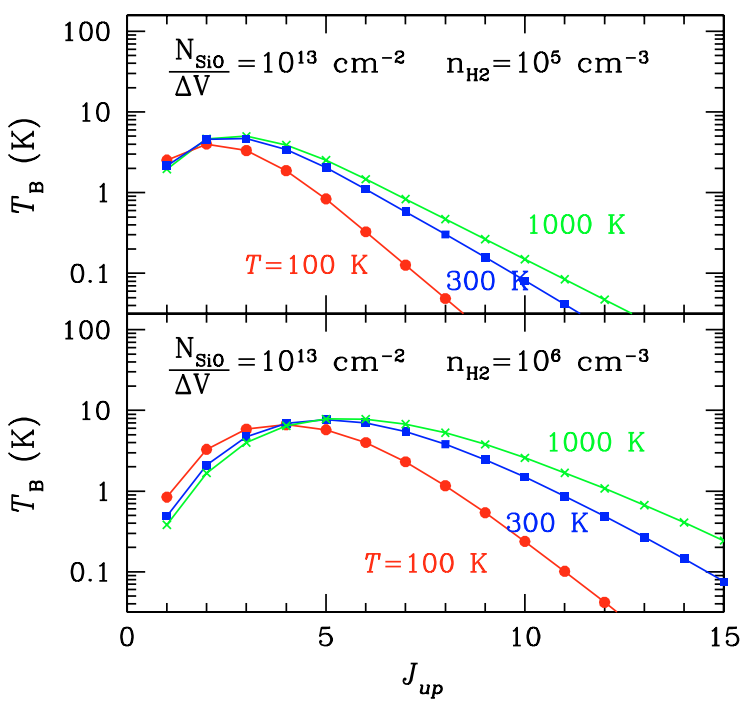

Fig. 5. SiO brightness temperatures predicted by LVG model calculations for different temperatures and for densities of $10^{5} \mathrm{~cm}^{-3}$, (upper panel) and $10^{6} \mathrm{~cm}^{-3}$ (lower panel). A ratio between column density and velocity spread $N_{\mathrm{SiO}} / \Delta V$ equal to $10^{13} \mathrm{~cm}^{-2} \mathrm{~km}^{-1} \mathrm{~s}$ has been considered in this plot.

with total velocity about $10 \mathrm{~km} \mathrm{~s}^{-1}$. Since this value is significantly larger than the typical $\mathrm{SiO}$ column densities derived in shocked regions (i.e. Codella et al. 1999), we have assumed in the first instance that the lines are optically thin in order to derive temperature and density from the different line ratios. Large values of $N_{\mathrm{SiO}} / \Delta V$ have been introduced only when a reasonable fit could not be obtained assuming optically-thin emission. Once $T$ and $n_{\mathrm{H}_{2}}$ have been estimated, the $\mathrm{SiO}$ column density has been derived from the absolute intensity of the $J=5-4$ line. We selected this line because is the one with the smaller beam having good $\mathrm{S} / \mathrm{N}$ ratios. From the derived column density and the velocity dispersion measured on our spectra, we have checked a posteriori the validity of our assumption of low opacity.

We have applied the LVG code on the intensities of each of the observed bullet, integrated in all the velocity channels, separating only the blue-shifted and red-shifted components. The results of the LVG analysis are discussed below for L1448 and L1157. 


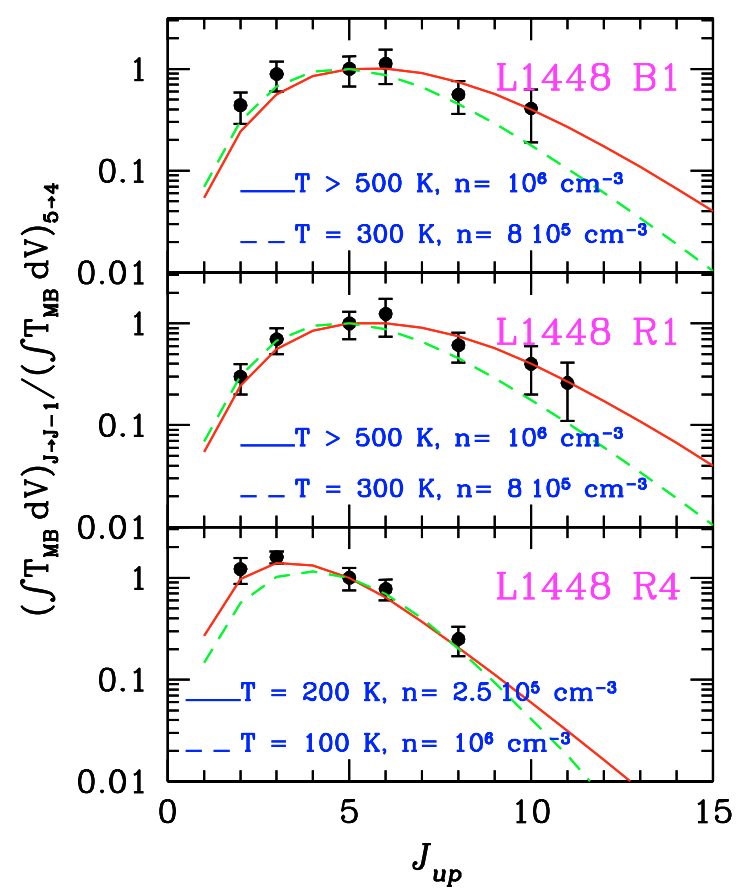

Fig. 6. SiO line intensities observed in knots $\mathrm{B} 1, \mathrm{R} 1$ and $\mathrm{R} 4$ of the L1448 outflow, normalized to the $J=5-4$ intensity and compared with the LVG model computations. Solid lines refer to the best fit through the data, while the dashed line represents the model fit at the lowest temperature which is still compatible with the data errors. The plotted line ratios uncertainty consider both the individual rms errors and an absolute calibration uncertainty of $10 \%$ for the lines with $J \leq 8$ and $20 \%$ for the $J=10$ and 11 lines.

\subsubsection{L1448}

Figure 6 shows the observed integrated intensities, normalised to the 5-4 transitions, of the bullets sampled by our observations, compared with the LVG model computations. Errors on line ratios take into account both the rms in the spectra and the calibration uncertainties. Assuming a velocity dispersion of $20 \mathrm{~km} \mathrm{~s}^{-1}$, i.e. comparable to the FWHM of the observed lines, the modelled line ratios are insensitive to the column density up to values $10^{15} \mathrm{~cm}^{-2}$. Since our observations do not fully sample the region covered by the larger $2-1$ beam, the applied convolution may not properly take into account all the emission region. In addition to this, the lack of a good fit may signal the presence of gradients in the physical parameters, with components at lower density/temperature encompassed by the large beams. Consequently, we adopted the best model that fits only the lines with $J \geq 5$, which have been obtained with a more similar beam size and thus trace similar gas components. A density of $n_{\mathrm{H}_{2}}=$ $10^{6} \mathrm{~cm}^{3}$ is well constrained by the observed ratios, while any temperature $T \gtrsim 500 \mathrm{~K}$ gives an equally good fit. We have also considered models assuming a higher density and lower temperature to explore whether other pairs of values can fit the data equally well. We find that within the errors, the line ratios of knots B1 and R1 can be consistent with a temperature of $300 \mathrm{~K}$ (see Fig. 5).

In the bullet R4 the best fit is obtained for $T=200 \mathrm{~K}$ and $n_{\mathrm{H}_{2}}=2.5 \times 10^{5} \mathrm{~cm}^{3}$ consistent with the lower excitation nature of this clump with respect to B1 and R1.

$\mathrm{SiO}$ column densities, determined from the absolute observed intensity of the 5-4 line, are of the order of 3-3.5 $\times$ $10^{13} \mathrm{~cm}^{-2}$ in all three bullets. Values derived for the B1 and R1 clumps, however, are probably just lower limits, since the

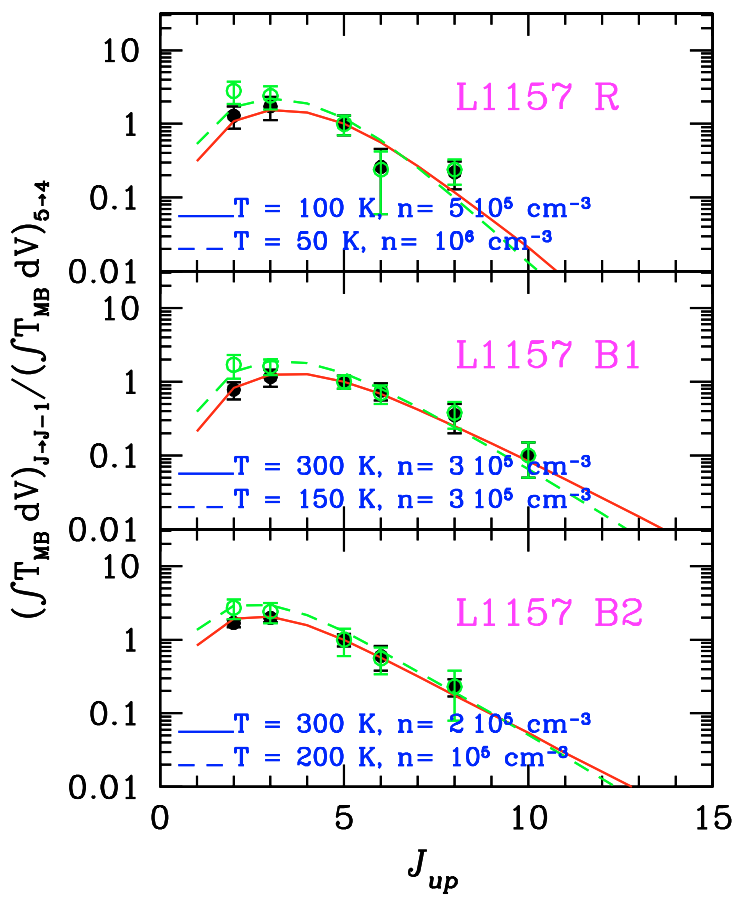

Fig. 7. The same as Fig. 8 but for the knots R, B1 and B2 of L1157. Filled circles refer to observed ratios assuming a beam filling of 1 ; open circles are the observed ratios corrected assuming an emitting size of $\sim 20^{\prime \prime}$ for R and B1, and 30" for B2 (see Sect. 3). The best fits obtained considering only the $J \leq 5$ lines (solid) and all the beam filling corrected lines (dashed) are reported.

emission region does not fill the entire 11 arcsec beam of the 5-4 observation. If the emission region is confined to a jet-like knot about $2^{\prime \prime}$ in diameter (see e.g. Guilloteau 1992), the corresponding column density would be enhanced by about a factor of 4 , thus being of the order of $10^{14} \mathrm{~cm}^{-2}$.

\subsubsection{L1157}

Figure 7 shows the observed line intensities, normalized to the $J=5-4$ transition, in the bullets R, B1 and B2, where we performed the LVG analysis. In the figure, filled circles represent line ratios not corrected for beam filling effects; open circles represent ratios corrected for beam filling, assuming the emission sizes estimated by convolving the $J=8-7$ observations to the $J=2-1$ beam (see previous section). This correction significantly affects only the 2-1 and 3-1 transitions, so we first fitted only the lines with $J \geq 5$. In B1 and B2 the line ratios are consistent with a temperature of $\sim 300 \mathrm{~K}$, a density $\sim 3 \times 10^{5} \mathrm{~cm}^{3}$ and optically-thin lines. The bullet $\mathrm{R}$, on the other hand, is consistent with colder gas at $T \sim 100 \mathrm{~K}$. These fits reproduce rather well the 2-1 and 3-2 uncorrected ratios. We have also tried to reproduce all the beam-filling-corrected data points, obtaining the fit indicated by dashed lines in Fig. 7. Good fits are obtained also by adopting slighter lower temperatures of $\sim 50-200 \mathrm{~K}$. Column densities are 2, 5 and $8 \times 10^{13} \mathrm{~cm}^{-2}$ at bullets R, B2 and B1 respectively. Therefore, the L1157 clumps appear less excited than the L1448 B1 and R1 bullets, confirming a dependence of the excitation conditions on the velocity of the bullets. 


\subsection{Excitation as a function of velocity}

The above LVG analysis was performed on intensities integrated over all the observed velocity channels, thus giving physical parameters averaged over all the emitting gas. The observed line profiles, however, are not symmetric and clearly show that gas components at different velocities are seen along the line of sight. We can then investigate whether these components are associated with different excitation conditions.

Figure 4 shows that in the L1157-B1 spectra there are at least two components, one peaking close to ambient velocity and one at about $-15 \mathrm{~km} \mathrm{~s}^{-1}$, whose relative $T_{B}$ ratio increases going from the $2-1$ to the $8-7$ line (see also Fig. 8). The signal-to-noise ratio in the line wings of the high- $J$ lines is not high enough to perform a separate LVG analysis in the different velocity channels. However we can still infer a quantitative trend in the excitation as a function of velocity by comparing sensitive line ratios. In Fig. 8 we have plotted the $\mathrm{SiO} 8-7 / 5-4$ ratio as a function of velocity for the L1157 B1 bullet and for the L1448 R1 and B1 bullets. The 8-7 and 5-4 lines have been observed with similar beamsizes and should thus not be much affected by different beam fillings.

For L1157-B1 the 8-7/5-4 ratio is $\sim 0.25 \pm 0.03$ in the peak close to ambient $V_{\mathrm{LSR}}$ velocity and $\sim 0.6 \pm 0.25$ at velocities $\sim-15 \mathrm{~km} \mathrm{~s}^{-1}$. In L1448 B1/R1, line ratio differences with velocity are less clear, although a trend is still recognisable in the B1 bullet, where the 8-7/5-4 ratio varies from $\sim 0.2-0.3 \pm 0.2$ at $V_{\mathrm{LSR}} \sim-20 \mathrm{~km} \mathrm{~s}^{-1}$ and $\sim 0.8 \pm 0.08$ at $V_{\mathrm{LSR}} \sim-50 \mathrm{~km} \mathrm{~s}^{-1}$. A ratio $\sim 0.2-0.3$ is consistent with gas at a density of $\sim 10^{6} \mathrm{~cm}^{3}$ and temperature $\sim 200 \mathrm{~K}$, while a value of $0.5-0.7$ would require either a higher temperature $(>500 \mathrm{~K})$ or a higher density $(\sim 5 \times$ $\left.10^{6} \mathrm{~cm}^{3}\right)$.

In L1448 B1/R1 the observed excitation as a function of velocity is consistent with gas coming from different layers behind a shock: the maximum temperature is found at the shock front, where the gas is compressed and the velocity is close to the shock velocity, and lower temperatures occur further out where the gas has been slowed down. In L1157-B1, however, we find a puzzling result, since most of the gas emission comes from the lower excitation component close to the ambient velocity, a feature that is not predicted by shock models, as we will further discuss in Sect. 4. Jiménez-Serra et al. (2004) have suggested that $\mathrm{SiO}$ emission at ambient velocity associated with outflows could be a signature of the interaction between the magnetic shock precursor in a C-type shock, with the ambient pre-shock gas. This interpretation in the case of L1157-B1 is in contrast to the large $\mathrm{SiO}$ abundance of the low velocity component. The derived velocity averaged column density of $8 \times 10^{13} \mathrm{~cm}^{-2}$ is almost entirely dominated by the gas component at ambient velocity. Such a high column density implies that this component should have experienced significant shock reprocessing. In addition, the ambient component due to the precursor is expected to have very narrow widths, of the order of $1 \mathrm{~km} \mathrm{~s}^{-1}$ or less, as observed by Jiménez-Serra et al. (2004) in L1448, while in L1157 the width of the component at ambient velocity is $\sim 7 \mathrm{~km} \mathrm{~s}^{-1}$. The inferred different excitation conditions of the low and high-velocity components in L1157 instead could be due to an evolutionary effect, where the $\mathrm{SiO}$ gas at ambient velocity in L1157 arises from decelerated post-shocked material that has already slowed down, as suggested in Codella et al. (1999).

The velocity structure of the L1448 B1 and R1 bullets, coupled with the inferred variations of physical parameters with the velocity, resemble very closely those found in optical/IR jetknots near the protostar. Indeed, the base of the optical jets

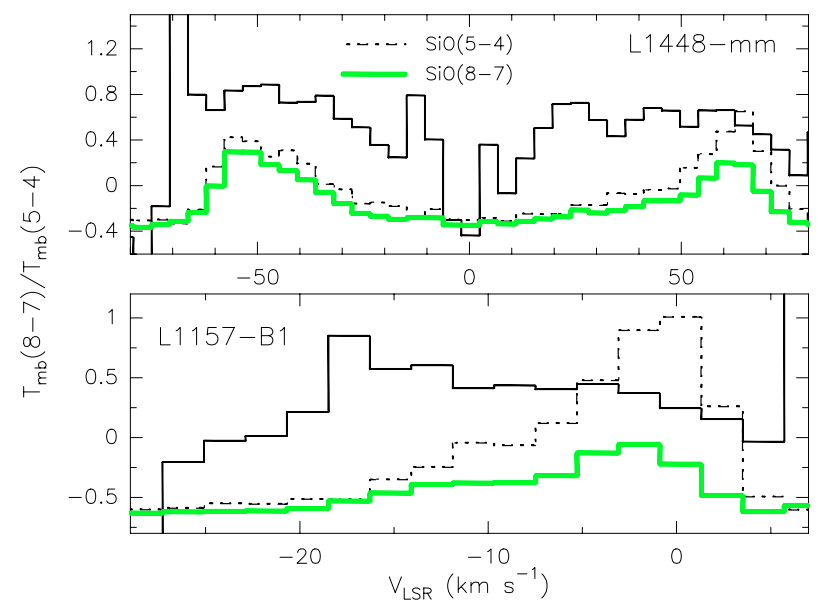

Fig. 8. Comparison between the intensity profiles vs. velocity of the SiO 5-4 and 8-7 lines in the L1448 R1 and B1 bullets (upper) and the L1157 B1 bullet (lower). The dot-dashed and grey lines are the individual profiles of the 5-4 and 8-7 lines, respectively, while the black line represents the profile of the line ratio. There is a trend, more evident for L1157 B1 and L1448 B1, showing that this ratio increases with the velocity.

structure is characterised by the presence of both low and high velocity components, with this latter being the brightest and having the highest excitation conditions (e.g. Ray et al. 2006). This similarity, and the evidence that the collimated EHV bullets are hotter and denser than usually found in the $\mathrm{CO}$ entrained outflow, suggest that they may indeed trace the main jet from the embedded protostar. Such an interpretation has been also suggested for the EHV $\mathrm{SiO}$ jet of HH211 on the basis of its excitation and velocity structure derived from SMA observations (Hirano et al. 2006).

\section{Comparison with other warm gas tracers}

In the physical conditions traced by the $\mathrm{SiO}$ emission, i.e. $T \sim$ $100-500 \mathrm{~K}$ and $n \sim 10^{5}-10^{6} \mathrm{~cm}^{-3}$, the main gas coolants are pure-rotational $\mathrm{H}_{2}$ lines, $\mathrm{CO}$ transitions with $J_{\text {up }}$ between 10 and 20 , and $\mathrm{H}_{2} \mathrm{O}$ rotational lines. $\mathrm{H}_{2} \mathrm{O}$ cooling is only significant if the conditions are appropriate to have large water abundances; this is indeed the case in low-velocity C-type shocks where most of the gas-phase $\mathrm{SiO}$ is expected to be produced. Most of the cooling from these molecules occurs at mid and far IR wavelengths. Since both the investigated outflows have been observed with ISO we can compare our diagnostic of the $\mathrm{SiO}$ emission with the results obtained by ISO for the other main coolants (see Table 4). In L1448, strong $\mathrm{CO}$ with $J>14$ and $\mathrm{H}_{2} \mathrm{O}$ emission is associated with the $\mathrm{B} 1$ and $\mathrm{R} 1$ bullets and the inferred physical conditions are $T=1200 \mathrm{~K}$ and $n_{\mathrm{H}_{2}}=6 \times 10^{4} \mathrm{~cm}^{-3}$ (Nisini et al. 1999, 2002). The temperature is also consistent with the $\mathrm{H}_{2} \mathrm{O}-0$ lines detected by SWS. The $\mathrm{SiO}$ emission seems to be confined to a denser region than for the other coolant, which implies density stratification inside the bullets. In the R4 bullet the conditions derived from the ISO-observed CO lines are $n_{\mathrm{H}_{2}} \sim$ $1-3 \times 10^{4} \mathrm{~cm}^{-3}$ and $T \sim 600 \mathrm{~K}$. These are consistent with the lower excitation found in this position with $\mathrm{SiO}$, although for this a higher density and lower temperature are estimated. From the comparison of our derived $\mathrm{SiO}$ column densities an the $\mathrm{N}(\mathrm{CO})$ estimated from the ISO data $\left(\sim 10^{17} \mathrm{~cm}^{-2}\right.$, Nisini et al. 2002), we obtain a SiO abundance of $\sim 10^{-7}$ and $3 \times 10^{-8}$ at the B1/R1 and $\mathrm{R} 4$ positions (Table 5 ). 
Table 4. Physical parameters of the observed bullets.

\begin{tabular}{lccccc}
\hline \hline & \multicolumn{2}{c}{$\mathrm{SiO}$} & \multicolumn{2}{c}{$\mathrm{CO}(J>14)^{a}$} & $\mathrm{H}_{2} 0-0^{b}$ \\
& $\begin{array}{c}T_{\text {kin }} \\
\mathrm{K}\end{array}$ & $\begin{array}{c}n_{\mathrm{H}_{2}} \\
\mathrm{~cm}^{-3}\end{array}$ & $\begin{array}{c}T_{\text {kin }} \\
\mathrm{K}\end{array}$ & $\begin{array}{c}n_{\mathrm{H}_{2}} \\
\mathrm{~cm}^{-3}\end{array}$ & $\begin{array}{c}T_{\text {kin }} \\
\mathrm{K}\end{array}$ \\
\hline & \multicolumn{5}{c}{$\mathrm{L} 1448$} \\
$\mathrm{~B} 1$ & $>500$ & $8 \times 10^{5}$ & 1200 & $6 \times 10^{4}$ & $950-1900$ \\
$\mathrm{R} 1$ & $>500$ & $10^{6}$ & 1200 & $6 \times 10^{4}$ & $950-1900$ \\
$\mathrm{R} 4$ & 200 & $2.5 \times 10^{5}$ & $600-650$ & $1-3 \times 10^{4}$ & $600-650$ \\
\hline \multicolumn{7}{c}{$\mathrm{L} 1157$} \\
$\mathrm{~B} 1$ & $150-300$ & $3 \times 10^{5}$ & $350-800$ & $5-60 \times 10^{5}$ & $560-1040$ \\
$\mathrm{~B} 2$ & $200-300$ & $2 \times 10^{5}$ & $350-800$ & $5-60 \times 10^{5}$ & $560-1040$ \\
$\mathrm{R}$ & $50-100$ & $1-5 \times 10^{5}$ & $\ldots$ & $\ldots$ & $570-860$ \\
\hline
\end{tabular}

${ }^{a}$ Derived from ISO-LWS data: Nisini et al. (2002) for L1448 and Giannini et al. (2001) for L1157. ${ }^{b}$ Derived from ISO-SWS and ISO-CAM data: Nisini et al. (2002) for L1448 and Cabrit et al. (1998) for L1157.

In L1157 the temperature and density estimated from the ISO data ranges between $350-800 \mathrm{~K}$ and $5 \times 10^{5}-6 \times 10^{6} \mathrm{~cm}^{-3}$ (Giannini et al. 2001), thus being fairly consistent with the parameters derived from $\mathrm{SiO}$. Temperatures in the same range are also found from the $\mathrm{H}_{2}$ 0-0 lines observed with ISOCAM (Cabrit et al. 1998). Therefore it appears that the physical conditions in the B1 bullets are more uniform, without strong gradients. In both $\mathrm{B} 1$ and $\mathrm{R}$ bullets, the $\mathrm{SiO}$ abundance is of the order of $6-8 \times 10^{-8}$.

From the comparison of the $\mathrm{SiO}$ column density and the column density of the warm water traced by ISO, we derive a $\mathrm{SiO} / \mathrm{H}_{2} \mathrm{O}$ abundance ratio of $\sim 2-3 \times 10^{-4}$ in L1448 and $\sim 10^{-3}$ in L1157 (see Table 5). The difference in these two values cannot be explained by the different excitation conditions of the two flows. For low velocity shocks, the $\mathrm{SiO} / \mathrm{H}_{2} \mathrm{O}$ ratio is sensitive to the shock speed, since shocks with velocity $\leq 20 \mathrm{~km} \mathrm{~s}^{-1}$ do not efficiently release silicon by sputtering of dust grains, while still being very efficient in producing large $\mathrm{H}_{2} \mathrm{O}$ abundances through high temperature $(T \gtrsim 300 \mathrm{~K})$ chemical reactions. However, both the maximum velocity and the excitation conditions observed in L1157 indicate that here the shock speed should be lower than in $\mathrm{L} 1448$. The different $\mathrm{SiO} / \mathrm{H}_{2} \mathrm{O}$ ratio observed could be due instead to an evolutionary effect, if the two molecules have different destruction timescales after the passage of the shock. The timescales for $\mathrm{H}_{2} \mathrm{O}$ depletion onto the dust grains range between $10^{4}-10^{5} \mathrm{yr}$ depending on the density (Bergin et al. 1998). Similar timescales are also predicted for the $\mathrm{SiO}$ destruction either by depletion on dust grains or by reactions with $\mathrm{OH}$ which leads to the formation of $\mathrm{SiO}_{2}$ (Codella et al. 1999; Schilke et al. 1998). Thus no simple considerations based on excitation conditions and evolutionary timescales seem to explain the different observed abundance ratio. More detailed chemical models of the two regions are required for a proper understanding of this point.

\section{Comparison with predictions from shock models}

The multi-line analysis performed can be used to check whether the derived excitation conditions and abundances are in agreement with the expectations from shock models.

The SiO molecule is produced in C-type shocks by the injection into the gas-phase of silicon by sputtering and/or graingrain collisions, followed by gas-phase reactions with $\mathrm{O}$ and $\mathrm{O}_{2}$ (Schilke et al. 1997; Caselli et al. 1997). To be efficient in producing the large observed column densities of $\mathrm{SiO}$ by sputtering,
Table 5. $\mathrm{SiO}$ and $\mathrm{H}_{2} \mathrm{O}$ abundances.

\begin{tabular}{lccc}
\hline \hline & $N(\mathrm{SiO})\left(\mathrm{cm}^{-2}\right)$ & $X(\mathrm{SiO})$ & $X(\mathrm{SiO}) / X\left(\mathrm{H}_{2} \mathrm{O}\right)^{a}$ \\
\hline L1448 B1-R1 & $\sim 10^{14}$ & $\sim 10^{-7}$ & $\sim 2 \times 10^{-4}$ \\
L1448 R4 & $3 \times 10^{13}$ & $3 \times 10^{-8}$ & $3 \times 10^{-4}$ \\
\hline L1157 B1 & $8 \times 10^{13}$ & $8 \times 10^{-8}$ & $10^{-3}$ \\
L1157 R & $2 \times 10^{13}$ & $6 \times 10^{-8}$ & $\sim 10^{-3}$ \\
\hline
\end{tabular}

a Assuming the $\mathrm{H}_{2} \mathrm{O}$ abundance derived by Nisini et al. (2002, L1448) and Giannini et al. (2001, L1157).

such shocks need to have speeds of at least $25 \mathrm{~km} \mathrm{~s}^{-1}$. At lower velocities $\left(V_{\mathrm{s}} \sim 20 \mathrm{~km} \mathrm{~s}^{-1}\right)$ and high density, grain-grain collisions may be more efficient than sputtering for the production of gas-phase elemental silicon. At such speeds, the neutral temperature of the post-shocked gas rises above $1000 \mathrm{~K}$ and then gently declines to return to its pre-shock values in a region of $\sim 5 \times 10^{16} \mathrm{~cm}\left(\sim 10^{\prime \prime}\right.$ at a distance of $350 \mathrm{pc}$, i.e. comparable with the bullet dimensions).

We can compare the observed line intensity distributions as a function of $J$ (Figs. 5 and 6 ) with those predicted by the Schilke et al. (1987) shock models as a function of pre-shock density and velocity (see their Fig. 6). The line ratios derived for the L1448 bullets are consistent with $n\left(\mathrm{H}_{2}\right) \sim 10^{6} \mathrm{~cm}^{-3}$ and shock velocities larger than $30 \mathrm{~km} \mathrm{~s}^{-1}$. L1157 B1 is instead better fit by a shock at lower density, between $10^{5}$ and $10^{6} \mathrm{~cm}^{-3}$. The models are not very sensitive to the shock velocity for $v_{\mathrm{s}}$ larger than $30 \mathrm{~km} \mathrm{~s}^{-1}$. This is a consequence of the fact that the post-shocked temperature is a function of the velocity, and that the $\mathrm{SiO}$ line ratios become insensitive to temperature variations for $T$ larger than $\sim 500 \mathrm{~K}$. However, the peak post-shock temperature reaches values larger than $1000 \mathrm{~K}$ in a $25 \mathrm{~km} \mathrm{~s}^{-1}$ shock (e.g. Kaufman \& Neufeld 1996), thus the $\mathrm{SiO}$ emission region is indeed expected to be very warm. Observations of submillimetre tracers with higher excitation energies, such as the high- $J$ CO lines, are needed to better constrain the temperature values inside the bullets.

The fractional $\mathrm{SiO}$ abundances derived in Table 5 are also consistent with those predicted by models in the regions of the post-shocked gas having the larger temperatures (between $10^{-8}$ and $10^{-7}$ ). In further out regions, where the temperature drops close to the pre-shock values, $\mathrm{SiO}$ is in part converted to $\mathrm{SiO}_{2}$ by reactions with $\mathrm{OH}$, and its abundance decreases to values below $10^{-9}$. The averaged high observed abundances therefore reveal that we are indeed observing the warmer regions just behind the shock front.

We can compare the observed line profiles with those predicted by models. Schilke et al. (1997) computed how the profiles change as a function of the rotational quantum number, both in the case of sputtering of Si-bearing material in grain cores and for the case of mantle sputtering. The overall profiles produced in the two cases are similar to those observed in the L1448 bullets: very asymmetric, with a gradual decrease of intensity for velocities lower than peak velocity and a very sharp decrease on the other side. In these bullets the intensity peaks occur at a $V_{\mathrm{LSR}}$ velocity of about $60 \mathrm{~km} \mathrm{~s}^{-1}$ which, deconvolved for inclination effects, corresponds to a total velocity greater than $150 \mathrm{~km} \mathrm{~s}^{-1}$ (for an inclination angle of $69^{\circ}$, Girart \& Acord 2001). This is much larger than the estimated shock velocity, implying that the bullet is probably moving in a medium that is already moving, and thus that the shock occurs at the much lower velocity given by the differential speed between the two fluids. 


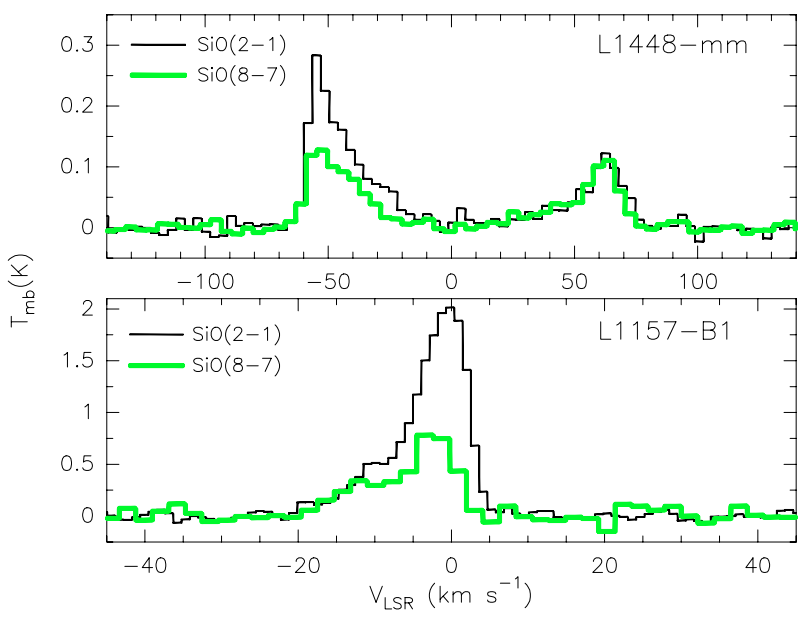

Fig. 9. Comparison between the intensity profiles of the $\mathrm{SiO} 2-1$ (black) and 8-7 (gray) lines in the L1448 R1 and B1 bullets (upper) and the L1157 B1 bullet (lower). The L1448 8-7 line profile toward the $\mathrm{mm}$ source central position has been convolved to the beam of the $2-1$ transition in order to compare the same emitting area.

In contrast with L1448, the L1157-B1 profiles do not appears consistent with the predicted ones, since the brightness peak is close to the ambient velocity and the intensity declines towards the higher velocity. We have already pointed out and discussed this behaviour in the previous sections, suggesting that the peak at ambient velocity could be due to a shock that has already slowed down but still retain the original $\mathrm{SiO}$ abundance. Models taking into account time evolution effects are needed to test this hypothesis. Geometrical effects can be also important and are expected to play a role in the line profile appearance. Bow shock models, for example, produce line profiles that are highly dependent upon the bow inclination angle and for some configurations produce shapes similar to those observed in the $\mathrm{L} 1157 \mathrm{SiO}$ line (see e.g. Schultz et al. 2005).

Another effect predicted by the Schilke et al. (1997) model is that the line profile broadens for lines with increasing $J$. Such a trend is not seen in our observed lines. In Fig. 8, for example, the profiles of the 2-1 and 8-7 lines are compared: in both the objects (L1448 and L1157) the two lines are equally broaded.

Thus, current shock models fit reasonably well the average physical conditions and abundances of the $\mathrm{SiO}$ gas derived from our multiline analysis in both the EHV bullets of L1448 and in the lower velocity clumps of L1157. However, the line profiles are still not well reproduced, especially when profile variations among lines with different excitation are taken into account. Effects such as a time-dependent chemistry and a geometry different from plane-parallel need to be taken into account for a more quantitative comparison with the observed profiles. In addition, interferometric observations should be able to spatially resolve the emitting regions contributing to the different components, thus allowing us to constrain the geometry to be adopted in a more detailed modelling. It would also be instructive to compare the observed $\mathrm{SiO}$ profiles with the profiles of other molecules similarily affected by a time dependent chemistry. $\mathrm{H}_{2} \mathrm{O}$, in particular, will be studied in detail with the Herschel observatory, providing valuable constraints on the shock model chemistry.

\section{Conclusions}

We have presented the first $\mathrm{SiO}$ multi-line analysis (from $J=$ $2-1$ to $J=11-10$ ) of the molecular bullets along the outflows of the Class 0 sources L1448-mm and L1157-mm. In L1448-mm, the bullets represent collimated and regularly spaced clumps travelling at velocities of hundreds of $\mathrm{km} \mathrm{s}^{-1}$. In contrast, the L1157 bullets have lower velocities and are characterised by a rich chemistry. From our analysis, we investigated the physical properties of these different types of bullets, exploring their excitation conditions and the ability of current models to reproduce the various $\mathrm{SiO}$ line ratios and profiles. The main results of our analysis are the following:

- In L1448, the SiO line ratios of the EHV molecular bullets $\mathrm{B} 1$ and R1 close to the driving source are consistent with a gas at $n_{\mathrm{H}_{2}} \sim 10^{6} \mathrm{~cm}^{-3}$ and $T_{\text {kin }} \gtrsim 500 \mathrm{~K}$. Conversely, the bullet R4, located at the apex of the red lobe molecular outflow and very strong in the $\mathrm{SiO} 2-1$ emission, is associated with lower excitation gas $\left(n_{\mathrm{H}_{2}}=2.5 \times 10^{5} \mathrm{~cm}^{-3}\right.$ and $T_{\text {kin }}=$ $200 \mathrm{~K})$. The low velocity bullets in L1157 show lower excitation conditions, with $n_{\mathrm{H}_{2}} \sim 1-5 \times 10^{5} \mathrm{~cm}^{-3}$ and $T_{\text {kin }} \sim$ $100-300 \mathrm{~K}$. In both the sources, there is clear evidence of the presence of velocity components having different excitation conditions, with the denser and/or warmer gas associated with the gas at the higher speed.

- While in L1448 the bullets emission is dominated by the highly excited and high velocity gas, in L1157 the peak of the emission at the ambient velocity is associated with the lower excitation conditions. Such a difference could be due to evolutionary effects, where the $\mathrm{L} 1157 \mathrm{SiO}$ gas at ambient velocity is due to an older shock that has already slowed down and is progressively reaching the physical conditions of the ambient medium.

- The physical conditions averaged over all the velocity components are consistent with those inferred by means of midand far-IR lines observed with the ISO satellite. In L1448, however, the high- $J$ CO lines detected by ISO-LWS trace a gas with a density about an order of magnitude lower than that measured with the $\mathrm{SiO}$ lines, indicating that gradients in the physical parameters can be present in the bullet regions.

- The column density of the $\mathrm{SiO}$ gas does not show large variations in the different bullets, ranging between $2 \times 10^{13}$ and $10^{14} \mathrm{~cm}^{-2}$. A comparison to the ISO-LWS derived abundance of $\mathrm{H}_{2} \mathrm{O}$, a molecule also very sensitive to the shock conditions, shows that the $\mathrm{SiO} / \mathrm{H}_{2} \mathrm{O}$ abundance ratio is $\sim 2-3 \times 10^{-4}$ in L1448 and $\sim 10^{-3}$ in L1157. Such a different ratio is not easily explained by either the local excitation conditions or the different flow evolutionary timescales.

- Current shock models for the $\mathrm{SiO}$ excitation reproduce quite well the observed line ratios when shocks with velocities larger than $\sim 25 \mathrm{~km} \mathrm{~s}^{-1}$ are considered. The models however fail to predict the observed profile variations as a function of the line excitation, as well as the strong emission at ambient velocity observed in L1157. Effects such as a time-dependent chemistry and a geometry different from plane-parallel need to be taken into account for a more quantitative comparison with the observed profiles.

- The velocity structure and the variations of physical parameters with the velocity observed in the EHV bullets in L1448 resemble very closely those found in optical/IR jet-knots near the protostar, suggesting that the $\mathrm{mm}$ collimated bullets have a similar origin and excitation mechanism. 
Acknowledgements. B.N., T.G. and C.C. wish acknowledge support through the Marie Curie Research Training Network JETSET (Jet Simulations, Experiments and Theory) under contract MRTN-CT-2004-005592. The James Clerk Maxwell Telescope is operated by The Joint Astronomy Centre on behalf of the Particle Physics and Astronomy Research Council of the United Kingdom, The Netherlands Organisation for Scientific Research, and the National Research Council of Canada.

\section{References}

Arce, H. G., \& Goodman, A. A. 2001, ApJ, 554, 132

Arce, H. G., \& Goodman, A. A. 2002, ApJ, 575, 928

Bachiller, R., Martín-Pintado, J., Tafalla, M., Cernicharo, J., \& Lazareff, B. 1990, A\&A, 231, 174

Bachiller, R., Peréz Gutiérrez, M., Kumar, M. S. N., \& Tafalla, M. 2001, A\&A, 372,899

Bergin, E. A., Melnick, G. J., \& Neufeld, D. A. 1998, ApJ, 499, 777

Bourke, T. L., Garay, G., Lehtinen, K. K., et al. 1997, ApJ, 476, 781

Cabrit, S., Bontemps, S., Lagage, P. O., et al. 1998, in The Universe as seen by ISO, ed. P. Cox, \& M. F. Kessler, 449

Caselli, P., Hartquist, T. W., \& Havnes, O. 1997, A\&A, 322, 296

Chandler, C. J., \& Richer, J. D. 2001, ApJ, 555, 139

Codella, C., Bachiller, R., \& Reipurth, B. 1999, A\&A, 343, 585

Dutrey, A., Guilloteau, S., \& Bachiller, R. 1997, A\&A, 317, L55

Giannini, T., Nisini, B., \& Lorenzetti, D. 2001, ApJ, 555, 40
Girart, J. M., \& Acord, J. M. P. 2001, ApJ, 552, L63

Gibb, A. G., Richer, J. S., Chandler, C. J., \& Davis, C. J. 2004, ApJ, 603, 198 Gueth, F., \& Guilloteau, S. 1999, A\&A, 343, 571

Guilloteau, S., Bachiller, R., Fuente, A., \& Lucas, R. 1992, A\&A, 265, L49

Hatchell, J., Fuller, G. A., \& Ladd, E. F. 1999, A\&A, 346, 278

Hirano, N., Liu, S., Shang, H., et al. 2006, ApJ, 636, L141

Jiménez-Serra, I., Martín-Pintado, J., Rodríguez-Franco, A., \& Marcelino, N. 2004, ApJ, 603, L49

Kaufman, M. J., \& Neufeld, D. A. 1996, ApJ, 456, 61

Martín-Pintado, J., Bachiller, R., \& Fuente, A. 1992, A\&A, 254, 315

Nisini, B., Benedettini, M., Giannini, T., et al. 1999, A\&A, 350, 529

Nisini, B., Benedettini, M., Giannini, T., et al. 2000, A\&A, 360, 297

Nisini, B., Codella, C., Giannini, T., \& Richer, J. S. 2002, A\&A, 395, L25

Palau, A., Ho, P. T. P., Zhang, Q., et al. 2006, A\&A, 636, L137

Richer, J. S., Shepherd, D. S., Cabrit, S., Bachiller, R., \& Churchwell, E. 2000, in Protostars and Planets IV, ed. V. Mannings, A. P. Boss, \& S. S. Russel (Tucson: Univ. Arizona Press), 867

Schilke, P., Walmsley, C. M., Pineau des Forêst, G., \& Flower, D. R. 1997, A\&A, 321,293

Schöier, F. L., van der Tak, F. F. S., van Dishoeck, E. F., \& Black, J. H. 2005 , A\&A, 432, 369

Schultz, A. S. B., Burton, M. G., \& Brand, P. W. J. L. 2005, MNRAS, 358, 1195

Tafalla, M., Santiago, J., Johnstone, D., \& Bachiller, R. 2004, A\&A, 423, L21

Turner, B. E., Chan, K. W., Green, S., \& Lubowich, D. A. 1992, ApJ, 399, 114

van Dishoeck, E. F., \& Blake, G. A. 1998, ARA\&A, 36, 317

Viti, S., Codella, C., Benedettini, M., \& Bachiller, R. 2004, MNRAS, 350, 1029 